\title{
Redes sociales y Tecnologías de la Información y la Comunicación en Educación: aprendizaje colaborativo, diferencias de género, edad y preferencias
}

\author{
Social networks and Information and Communications Technology in \\ Education: collaborative learning, gender differences, age and preferences
}

\author{
Julio Cabero Almenara \\ Universidad de Sevilla. España \\ cabero@us.es \\ Julio Barroso Osuna \\ Universidad de Sevilla. España \\ jbarroso@us.es \\ María del Carmen Llorente Cejudo \\ Universidad de Sevilla. España \\ karen@us.es \\ Cristina Yanes Cabrera \\ Universidad de Sevilla. España \\ yanes@us.es
}

\begin{abstract}
Resumen
Las redes sociales despiertan un inusitado interés social, profesional y académico. La carencia y necesidad de estudios e investigaciones que ofrezcan resultados y conclusiones a la posible relación entre el uso de las Redes Sociales y su vinculación con el trabajo colaborativo y otras variables (género, edad, país donde se cursan los estudios universitarios, entre otras), son el detonante del estudio que se presenta en el artículo, y cuyos propósitos generales están destinados a conocer si existen diferencias significativas entre el uso de éstas y dichas variables. Además, se pretende conocer qué herramientas sociales prefieren los discentes en su práctica didáctica. El instrumento empleado para llevar a cabo el trabajo es el elaborado por Anderson et al. (2009) denominado "Social Software survey used with unpaced undergrad". Algunos de los resultados encontrados muestran que sí existen diferencias significativas en cuestiones de género, mostrando las mujeres mayores habilidades en movilización de herramientas y los hombres en habilidades técnicas-tecnológicas, así como diferencias con respecto a la edad, entre otras.
\end{abstract}

\section{Palabras clave}

Redes sociales, trabajo colaborativo, enseñanza superior, teorías del aprendizaje, género.

\begin{abstract}
Social networks arouse an unusual social, professional, and academic interest. The lack and need for studies and research that provide results and conclusions to the possible relationship between the use of social networks and their links with collaborative work and other variables, inter alia: gender, age, country where university studies are made, etc., are the trigger of the study presented in the article, and whose general purposes are intended to determine whether there are significant differences between the use of all these variables. In addition, it aims to identify social tools learners prefer in their teaching practice. The instrument used to carry out the work is prepared by Anderson et al. (2009) called "Social Software survey used with unpaced undergrad". Some of the results found out show that there are significant differences in gender, so women show greater skills in tools mobilizing and men in technical-technological skills, as well as differences with respect to age, among others.
\end{abstract}


Key words

Social networking, collaborative work, higher education, learning theories, gender.

\section{Introducción}

En la sociedad actual, no cabe la menor duda que las redes sociales despiertan un inusitado interés social y profesional. Así como en el ámbito académico el interés se centra en conocer los efectos que éstas provocan en el éxito y desarrollo educativo de cualquier estudiante -más si cabe, niveles universitarios- (Abramson, 2011), en el resto de ámbitos, éstas se han convertido en grandes potenciales tecnológicos, ofreciendo una gran variedad de servicios para sus usuarios, brindando la posibilidad de ver videos, de reencontrarse con familiares perdidos desde hace décadas, de facilitar una manera de comunicarse, y de ser capaces de derribar las paredes físicas para fomentar la construcción de un sentido de "comunidad global", entre otras.

A través del concepto denominado como la “webvolución” (Kapp y O’Driscoll, 2010), es decir, la evolución de la web, el enfoque ha ido transformándose de "acceso y hallazgos" (Web 1.0), a "compartir, participar y colaborar" (Web 2.0), hasta llegar a la "colaboración inmersiva y co-creación" (Web 3.0) (Domínguez y Llorente, 2009; Llorente, 2013a). Es así como, a medida que se avanza hacia entornos comunicacionales de aprendizaje y colaboración, el interés en el uso educativo de las herramientas que las incorporan ha ido creciendo de manera exponencial. Pero, para ser justos en su conceptualización, cabe señalar que las redes sociales también son fruto de la evolución de otras herramientas que han ido transformándose, tal como puede comprobarse con las denominadas comunidades de prácticas o de aprendizaje en el terreno educativo (Martínez, 2003; Salinas, 2004; Cabero, 2006: Rodríguez Illeras, 2007; Cabero y Llorente, 2010), las cuales hacían referencia a la significación que tienen para el aprendizaje las acciones de trabajo colaborativo, cooperativo y grupal, y cuyas características más distintivas se apuntaban como:

- Accesibilidad para que todos los miembros puedan participar a todos los niveles (recibir, mandar mensajes, etc.). Ello no significa que la comunidad virtual no esté moderada y coordinada por el profesor, estableciendo diferentes perfiles y privilegios.

- Asumir una cultura de participación, colaboración y sentimiento de comunidad.

- Necesidad de mínimas competencias tecnológicas.

- Objetivos y fines claramente definidos y conocidos por todos sus miembros.

- Calidad de la información y contenidos relevantes (aunque ello dependerá de las características de los participantes y del papel del profesor).

- Motivación y compromiso.

- Normas y reglas de funcionamiento claras y conocidas por todos los miembros.

- Participación de todos sus miembros, es decir, gran presencia social.

- Disminución de la distancia transaccional, que es el espacio personalizado de comunicación y psicológico entre los estudiantes y el profesor (depende del control que ejerza el profesor y de la capacidad de diálogo y participación del alumno, a más control mayor distancia, a más diálogo menor distancia).

Redes sociales y Tecnologías de la Información y la Comunicación en Educación: aprendizaje colaborativo, diferencias de género, edad y preferencias. María del Carmen Llorente, Julio Cabero y Julio Barroso.

Página 2 de 23 
- Una estructura que potencie un espacio de interacción significativo y de confianza que fomente la interacción.

- Estar planificada y poseer un método para trabajar y llegar a acuerdos.

- Creación de un clima de creatividad, innovación e indagación.

En esta introducción, bien estará señalar que respecto a su definición existen diferentes perspectivas; las que se centran en la comunicación y la colaboración como variables fundamentales; o las que se basan en el estudio de las características estructurales de sus herramientas (Cabero, Barroso, Llorente y Marín, 2013). Por aportar alguna definición, citar la De Haro (2010), que las define como una estructura social que se puede representar mediante nodos conectados por aristas, donde los nodos representan a los individuos y las aristas las relaciones entre ellos. Otros autores, como Boyd y Ellison (2008), llaman la atención respecto a que son herramientas que facilitan que las personas construyan un perfil público o semipúblico dentro de un sistema delimitado, que les permite articular una lista de diferentes usuarios con los que comparte una conexión, y además poder ver las conexiones que suceden dentro de su sistema. Finalmente, señalar que para Cabero (2012), más centrado en una perspectiva de utilización educativa de las redes sociales, señala que son un multientorno que permiten la discusión de diferentes tipos de problemas en espacios interactivos y flexibles de aprendizaje; a ello posiblemente se le deba incorporar la característica de que permite ofrecer diferentes tipos de materiales a los estudiantes.

Respecto a su tipología, se encuentran con diferentes tipos: verticales-horizontales, profesionales-de ocio, abiertas-cerradas, gratuitas-de pago, libres-alojadas en servidores propios,... (De Haro, 2010), que implican posibilidades diferentes a las de su puesta en acción.

Para finalizar esta introducción, bien estará con realizar algunos comentarios respecto a sus posibilidades y limitaciones en su aplicación a la enseñanza. Por lo que se refiere a sus posibilidades, se pueden señalar un número de ellas de acuerdo con la propuesta formulada por diferentes autores (Cachia, 2008; Ortega y Gacitúa, 2008; Santamaría, 2008; Camacho, 2010; De Haro, 2010; Romero, 2011; Joosten, 2012; Cabero y Marín, 2013): permiten la creación natural de una inteligencia colectiva, amplían las fronteras del proceso de enseñanza aprendizaje, facilitan la comunicación, favorecen la construcción colaborativa del conocimiento, implican un cambio en la gestión de los procesos de enseñanza, fomentan una visión compartida del conocimiento, favorecen la socialización del grupo, son espacio de encuentro entre los diferentes actores del proceso enseñanza aprendizaje; permiten centralizar en un único sitio todas las actividades docentes, aumentan la fluidez y sencillez de la comunicación entre profesores y alumnos, facilitan la coordinación y trabajo de diversos grupos de aprendizaje, aumentan la interacción entre profesores y alumnos, facilitan la retroalimentación de los estudiantes a tiempo, mejora el aprendizaje, favorece la creación de un aprendizaje activo, permiten recrear grupos de trabajo y de actividades socializadoras, son fáciles de incorporar a la enseñanza ya que los alumnos se encuentran familiarizado con ellas, y se genera en el estudiante un proceso de análisis y

Redes sociales y Tecnologías de la Información y la Comunicación en Educación: aprendizaje colaborativo, diferencias de género, edad y preferencias. María del Carmen Llorente, Julio Cabero y Julio Barroso.

Página 3 de 23 
síntesis reforzando su actitud crítica al estar o no estar de acuerdo con otras opiniones disponibles en la herramienta.

Y respecto a sus inconvenientes, los siguientes: tener precaución con la privacidad, su incorporación exige del profesorado crear actividades motivadoras para que no se conviertan en un distractor y en un elemento lúdico y de ocio, la falta de formación de los padres en estos aspectos podría traer desconfianza y poco control al momento de usarlas en otros entornos, y, finalmente, considerar el riesgo del acoso cibernético (Roig, 2011; Aydin, 2012).

\subsection{Impacto de las Redes Sociales en la sociedad}

Existe un hecho evidente, y es que las tecnologías han acelerado el ritmo de avance en todos los aspectos de nuestras vidas. Sin embargo, la comunidad educativa sigue en su lucha constante de décadas con el propósito de establecer el verdadero papel que estas innovaciones deben desempeñar para hacer más eficiente la enseñanza y el aprendizaje en las aulas. La mayoría de los alumnos acuden a sus clases conectados con las tecnologías portátiles y móviles, pero a menudo el uso -didáctico/curricular- de éstas es inexistente, e incluso se puede mirar con recelo. Quizás, uno de los ejemplos más evidentes en la actualidad es la falta de conexión entre una de las herramientas preferidas por los estudiantes (las redes sociales) y el uso que los docentes hacen de ellas. Es por ese motivo, por lo que en los últimos años se está advirtiendo desde el ámbito educativo que el gran reto de la incorporación de las Tecnologías de la Información y la Comunicación (TIC) es ir más allá de la utilización de las herramientas de comunicación y la búsqueda y selección de la información, y aprovechar todas las posibilidades que la Web 2.0 ha desplegado a través de las diferentes utilidades que han quedado categorizadas bajo el término de "Software Social". Parameswaran \& Whinston (2007) lo definen como:

"una categoría de aplicaciones y servicios que facilitan la acción colectiva y la interacción social en línea con un rico intercambio de información multimedia que genera evolución del conocimiento de manera global".

Blogs, wikis, redes sociales, Youtube, Slideshare, o Flickr, entre otras, son ejemplos de algunas herramientas que se emplean para compartir y colaborar en contextos educativos, sociales o de negocios. Si hay que otorgarle un atributo distintivo a este tipo de herramientas podría ser que el contenido es generado por el propio usuario, por lo que éste se caracteriza por su dinamismo, sus cambios frecuentes, y a menudo, impredecibles. Así pues, entre todas estas herramientas, es posible hallar gran variedad, unas más actuales y otras más en desuso, pero todas bajo la premisa de las siguientes características:

- La importancia y la necesidad que supone la participación para el desarrollo y buen funcionamiento.

- Noción de usuario como contribuyente y como informador.

- Afianzamiento de la noción de poder de las multitudes.

- Enriquecimiento de la experiencia de los usuarios.

Redes sociales y Tecnologías de la Información y la Comunicación en Educación: aprendizaje colaborativo, diferencias de género, edad y preferencias. María del Carmen Llorente, Julio Cabero y Julio Barroso.

Página 4 de 23 
A ello, podrían agregarse otra serie de aspectos que Boyd (2005) apuntaba como necesarios, y que se sintetizan en:

- Soporte de conversaciones entre individuos o grupos, que van desde los mensajes instantáneos en tiempo real hasta los espacios de colaboración en tiempo diferido.

- Soporte para la retroalimentación que permita a un grupo conocer las contribuciones de los otros participantes, y que lleva de forma implícita a la "reputación digital".

- Soporte a la red social para crear y conducir, de forma explícita, una expresión digital de las relaciones personales de un individuo y ayudarlo a adquirir nuevas relaciones.

Evidentemente, del gran abanico de herramientas disponibles bajo el término software social, se pueden encuadrar una gran variedad, tales como: blogs y wikis, marcadores sociales o de etiquetado social, de edición y construcción colaborativa de recursos, widgets y microcontenidos (López y Ballesteros, 2008; Llorente, 2013b). Pero si hay una que ha alcanzado un desarrollo exponencial frente al resto, no cabe la menor duda que, esas son las denominadas redes sociales.

Bartlett-Bragg (2006) definía las redes sociales como:

"una gama de aplicaciones que aumentan las interacciones del grupo y los espacios comunes para el intercambio de información y colaboración, relaciones sociales de usuarios en un entorno basado en la red".

Por otro lado, Boyd \& Ellison (2008) las definen como aquellos servicios basados en la red que permiten a los individuos: a) construir un perfil público o semi-público dentro de un sistema delimitado, b) articular una lista con usuarios con los que se comparte conexión, y c) ver y estar al tanto de la lista de conexiones propias y del resto de usuarios.

La mayoría de las redes sociales están basadas, casi de manera exclusiva, en el uso de la web, facilitando la interacción entre los usuarios a través de diferentes servicios (chat, voz, blogs, grupos de discusión, etc.). Resulta evidente, tal como apuntaba ya por entonces Santamaría (2008), que las redes sociales estaban revolucionando la forma en que nos comunicábamos y compartíamos la información con otros en nuestro entorno, así que en la actualidad, nadie duda de que forman parte de nuestra vida cotidiana.

\subsection{Educación, investigación y Redes Sociales: antecedentes}

En el ámbito educativo, O'Keefee y Clarke (2011) enfatizaban sobre cómo el creciente uso de las redes sociales estuvo acompañado por la preocupación de los padres sobre los posibles perjuicios que éstas acarreaban para el éxito académico de sus hijos. En este sentido, uno de los estudios pioneros en investigar el efecto que las redes sociales, y

Redes sociales y Tecnologías de la Información y la Comunicación en Educación: aprendizaje colaborativo, diferencias de género, edad y preferencias. María del Carmen Llorente, Julio Cabero y Julio Barroso.

Página 5 de 23 
más concretamente Facebook, tenía en el éxito académico fue el realizado por Kirschner y Kirpinski (2010), basándose en más de 200 encuestas cumplimentadas por estudiantes universitarios, obteniéndose algunos de los resultados que se señalan a continuación: aquellos estudiantes que utilizaban la red social empleaban menos tiempo estudiando que los que no la usaban; los participantes usuarios de la red social contaban con pocas habilidades de gestión del tiempo; la utilización de la herramienta posponía su tiempo de estudio, entre otras.

Asimismo, y en contraposición a ello, diferentes investigaciones y estudios han demostrado que las herramientas disponibles bajo el software social, más concretamente las redes sociales, ofrecen soporte y apoyo a las actividades educativas, generando interacción, colaboración, participación activa, distribución de la información y de los recursos disponibles, así como fomentar el pensamiento crítico de los estudiantes (Ajjan \& Hartahorne, 2008; Mason, 2006).

Por lo tanto, puede considerarse que el uso de las redes sociales en los contextos educativos y de instrucción también poseen un potencial didáctico significativo. Güzin y Koçak (2010) llevaron a cabo el diseño de un estudio para conocer cómo los usuarios utilizaban las redes sociales (Facebook) con propósitos educativos. Para ello, establecieron un modelo donde analizaban las siguientes variables e hipótesis:

Utilidad: definida como "el grado en que una persona cree que el uso de un sistema particular mejora su desempeño laboral" (Davis, 1989). O como "el grado en que una innovación se percibe como mejor que su precursora" (Rogers, 2003). En este sentido, las redes sociales ofrecen posibilidades tales como la comunicación, colaboración, intercambio de información y disfrute, lo cual se sugieren como factores importantes que influyen en la incorporación de la herramienta. Los autores la definen como "la percepción formada por la creencia de que el uso de un sistema particular mejora el rendimiento de los individuos mientras se afirma que esta innovación particular resulta mejor que sus precursoras" (Güzin y Koçak, 2010).

Facilidad de uso: utilización de las funciones de las herramientas sociales de manera sencilla para gestionar el contenido global de la misma sin emplear grandes esfuerzos por parte de los usuarios. Por ejemplo, combinación de habilidades técnicas con las competencias específicas que el usuario posee, como por ejemplo, el manejo fotográfico, carga y descarga de archivos, edición de perfiles, o el uso de los diferentes menús.

Influencia social: explicado en términos de opinión preconcebida sobre cómo otros pueden juzgan el comportamiento de una persona en particular. Debido a que las redes sociales son herramientas utilizadas por gran cantidad de usuarios, las normas sociales poseen un papel significativo en el uso de esta herramienta. La influencia social en el estudio es definida como la percepción de cómo los seres queridos van a reaccionar a la realización de una conducta, en este caso concreto, formando parte de un nuevo entorno social.

Redes sociales y Tecnologías de la Información y la Comunicación en Educación: aprendizaje colaborativo, diferencias de género, edad y preferencias. María del Carmen Llorente, Julio Cabero y Julio Barroso.

Página 6 de 23 
Facilitador de condiciones: acceso a los servicios de apoyo que la herramienta nos ofrece para facilitar y gestionar nuestras propias actividades.

Identidad común: percibida como uno de los factores determinantes en la motivación de los usuarios para participar y formar parte de las comunidades virtuales. En los entornos sociales son especialmente relevantes, puesto que las personas se socializan en grupos de intercambio de ideas, recursos, materiales, discusión y colaboración continua, por lo que las redes sociales facilitan a los usuarios a crear sus propios grupos, unirse a los existentes, etc.

Fines en el uso de las redes sociales: pueden ser utilizadas para diferentes fines, con diferentes intereses y propósitos. En el estudio se plantean tres grandes efectos del uso de las redes sociales: relaciones sociales, relación en actividades laborales y actividades personales.

Relaciones sociales: para hacer nuevos amigos, mantenimiento de ya existentes, y propiciar la comunicación entre éstos.

Relaciones laborales: usuarios con propósitos profesionales de las mismas características que pueden acceder a diferente información, compartir proyectos, materiales, recursos, tareas o ideas.

Actividad diaria: actividades de ocio y de tiempo libre, mantenerse actualizado sobre lo que sucede alrededor de su círculo social, divertirse, jugar o unirse a diferentes grupos de intereses.

Uso educativo: las redes sociales facilitan el aprendizaje informal debido a su papel activo en la vida cotidiana de los miembros que las componen. Además, se potencia el aprendizaje colaborativo, involucrando a las personas en el pensamiento crítico, la comunicación y la mejora de las habilidades de escritura. Asimismo, Lee \& McLoughlin (2008) afirman que las redes sociales son herramientas pedagógicas porque la gente puede emplearlas para apoyar la conectividad social, el descubrimiento y la colaboración, el intercambio de información, la creación de contenidos, la agregación de conocimientos e información, así como su posterior modificación. Es necesario investigar el uso educativo de las redes sociales en tres ejes: intercambio de comunicación, colaboración, y recursos/materiales.

Comunicación: actividades que permiten la comunicación con otros estudiantes y sus profesores, facilitando las discusiones en clase, anuncios, entrega de trabajos, etc.

Colaboración: las redes sociales proporcionan oportunidades para que los miembros se unan a nuevas redes, de manera que se generan espacios para el aprendizaje colaborativo. Los usuarios pueden intercambiar ideas, compartir información y trabajar juntos desde y para intereses comunes, ideas o necesidades.

Recursos/ material compartido: como los usuarios intercambian ideas e información a través de las redes sociales, permiten compartir los recursos, materiales, proyectos y

Redes sociales y Tecnologías de la Información y la Comunicación en Educación: aprendizaje colaborativo, diferencias de género, edad y preferencias. María del Carmen Llorente, Julio Cabero y Julio Barroso. 
documentos. Para ello desarrollan capacidades para subir videos y fotos, seguir enlaces a recursos o páginas, o acceder a material audiovisual.

Los resultados del estudio desarrollado por Roblyer et al. (2010), indican que los profesores y los estudiantes difieren ligeramente en los usos actuales y futuros de las redes sociales, como por ejemplo, Facebook. Resulta de particular interés la diferencia significativa entre la percepción del papel de esta herramienta como más social que educativa. Así pues, los estudiantes se muestran mucho más "abiertos" que los docentes a la idea de incorporar las redes sociales de manera instruccional en las facultad.

De la gran variedad de redes sociales existentes en la red, no cabe duda que la más popular para los estudiantes universitarios es Facebook, hecho que demuestran diferentes investigaciones que apuntan que entre el 85 y el $99 \%$ de los mismos la utilizan en su vida cotidiana (Hargittai, 2008; Jones y Fox, 2009). Asimismo, datos recientes recopilados por el Centro de Investigación Aplicada EDUCAUSE (ECAR), reflejan que, de una muestra de 36.950 estudiantes procedentes de 126 universidades americanas, el $90 \%$ de los alumnos utilizaban las redes sociales, y que el $97 \%$ de la muestra empleaba Facebook como red social de preferencia, señalando además que participaban todos los días de manera activa en la misma.

Por otro lado, Martín-Moreno (2004) en un estudio desarrollado sobre las redes sociales en la educación, apuntaban que las potencialidades más útiles en el terreno educativo se establecían por los siguientes motivos: incrementan la motivación, favorecen mayores niveles de rendimiento académico (puesto que el aprendizaje individual y grupal se retroalimentan), mejoran la retención de lo aprendido, potencian el pensamiento crítico, y multiplican la diversidad de los conocimientos y las experiencias que se adquieren.

\subsection{Teorías de aprendizaje, trabajo colaborativo y Redes Sociales.}

Es evidente que las TIC proporcionan herramientas para el desarrollo de actividades de colaboración y cooperación en la enseñanza, facilitando la interacción de los estudiantes desde una perspectiva constructivista vinculada, de manera inexorable, a la teoría de Vygotsky (1978), o dicho en otros términos, la importancia de prestar apoyo individual o andamiaje para facilitar el desarrollo cognitivo de los estudiantes durante el proceso de aprendizaje. Para ello, se entiende como andamiaje la ayuda proporcionada por un profesor, un experto, o los propios compañeros más capaces que le permite al sujeto resolver un problema de manera independiente. Andrew Carnegie, empresario de principios de siglo XX, comentó que es un gran avance en el desarrollo cuando aceptamos que otras personas pueden audarnos a hacer mejor nuestro trabajo (Maxwell, 2001). Según Hannafin, Land y Oliver (1999) las ayudas se pueden clasificar en cuatro tipos: conceptuales (orientación sobre el qué aprender), metacognitivas (orientación sobre cómo pensar durante el aprendizaje), procedimentales (orientación sobre cómo utilizar los recursos y las herramientas), y estrategias (orientación sobre los enfoques para resolver un problema). Se hace necesario desarrollar en los procesos de formación las competencias que permitan afrontar las necesidades de la sociedad actual y futura en

Redes sociales y Tecnologías de la Información y la Comunicación en Educación: aprendizaje colaborativo, diferencias de género, edad y preferencias. María del Carmen Llorente, Julio Cabero y Julio Barroso.

Página 8 de 23 
términos de innovación y creatividad, adaptación a los nuevos entornos tecnológicos, y en la capacidad de colaborar en entornos de diversidad profesional e intercultural.

La "ecología del aprendizaje", tal como sugiere Barron (2006), proporciona "un conjunto de contextos en los espacios físicos o virtuales que proporcionan oportunidades de aprendizaje". Y para los alumnos, establece que: los adolescentes participan al mismo tiempo en muchos lugares; que crean contextos de aprendizaje por sí mismos dentro y entre las ciudades y que los límites entre los escenarios pueden ser permeables. Así pues, esta última idea también podría relacionarse con el concepto de "aprendizaje distribuido", visión que de acuerdo con Mason \& Rennie (2008) supone que: a) los componentes del curso se distribuyen a través de múltiples medios; b) puede ser usado para aumentar los cursos tradicionales basados en la clase o para ofrecer cursos de educación a distancia así como crear su totalidad de cursos en línea; c) proporcionar a los estudiantes la flexibilidad, en términos de tiempo o lugar de estudio.

Otra teoría es la denominada "conectivismo" (Siemens, 2005), en la que se unen una serie de principios en los que se basan la teoría del caos, las redes, la complejidad y la auto-organización, y cuyos principios que le dan sustento son:

1. El aprendizaje y el conocimiento se apoyan en una diversidad de conceptos.

2. Aprendizaje y conocimiento requieren diversidad de partes para presentar el todo.

3. Aprendizaje es el proceso de conexión entre fuentes de información y nodos especializados.

4. El conocimiento puede residir en equipos no humanos, y el aprendizaje puede facilitarse con el uso de la tecnología.

5. Las conexiones deben cuidarse y mantenerse para facilitar el aprendizaje continuo.

6. Destreza para identificar conexiones entre ideas, campos de conocimiento y conceptos es una habilidad clave muy importante.

7. Las actividades de aprendizaje están orientadas a mantener el conocimiento actualizado.

8. La capacidad de saber más, es más crítica que aquello que se sabe en un momento dado.

9. La toma de decisiones en sí misma es un proceso de aprendizaje.

El último marco de referencia que le dan cobertura a la incorporación de las redes sociales a la educación es el denominado "trabajo colaborativo y/o cooperativo". Cabero (2003, 135) ya apuntaba a éste como: un recurso, una estrategia y metodología de instrucción asociada fundamentalmente a la formación de adultos, en la cual varían los roles tradicionales desempeñados por el profesor y el estudiante en una metodología tradicional de transmisor y receptor de la información, y se pasa a nuevos entornos donde los conocimientos se desarrollan de forma conjunta y colaborativa. No solo se colabora para aprender, sino que al mismo tiempo se aprende a colaborar.

Redes sociales y Tecnologías de la Información y la Comunicación en Educación: aprendizaje colaborativo, diferencias de género, edad y preferencias. María del Carmen Llorente, Julio Cabero y Julio Barroso.

Página 9 de 23 
El aprendizaje colaborativo mejora potencialmente el aprendizaje y el rendimiento de los alumnos, y aumenta la motivación de éstos para llevar a cabo tareas grupales, lo que contribuye, a su vez, al aumento de la autoestima así como el desarrollo de habilidades de trabajo en equipo. En este sentido, los alumnos aprenden a ofrecer y a recibir ayuda de otros, a compartir el conocimiento, y a resolver dudas propias desde otro punto de vista, lo que les facilita más probabilidades para estar involucrados en aprender a exteriorizar sus propias ideas y reflexionar sobre la interacción producida, mientras interactúan con el resto de compañeros.

Para finalizar los comentarios que se han realizado, destacar dos últimos comentarios: uno, que los alumnos muestran altos niveles de satisfacción cuando se utilizan en la enseñanza y adquieren niveles significativos de aprendizaje (Aydin, 2012; Irwin, Ball, Desbrow y Leveritt, 2012; Cabero \& Marín, 2013; Greenhow y Gleason, 2014; Güler, 2015), y dos, que se han llevado a cabo más estudios y reflexiones teóricas que investigaciones (Veletsianos y Navarrete, 2012; López, Vásquez, y Sánchez-Serrano, 2015).

Con el desarrollo y la incorporación de las herramientas de software social, los sujetos pueden interactuar unos con otros de la manera que mejor se crea oportuna. Más concretamente, las redes sociales son capaces de modificar una gran variedad de formas culturales. Una de las características más importantes del trabajo colaborativo a través de las redes sociales, es que los medios facilitan a los usuarios a estar conectados y a poder compartir el contenido que ellos mismos generan -a escala mundial-. Personas de diferentes lugares del mundo, por ejemplo, pueden trabajar juntos en una actividad de escritura colaborativa en wikis para producir un "texto social". Es decir, y en otras palabras, las redes sociales proporcionan a los sujetos una plataforma de integración de conocimientos que pueden apoyar de una manera significativa el aprendizaje colaborativo.

\section{La investigación realizada.}

La carencia y escasez de estudios e investigaciones que ofrezcan resultados y conclusiones a la posible relación entre el uso de las Redes Sociales y su vinculación con el trabajo colaborativo y otras variables, fue el detonante a partir del cual se considera la necesidad de realizar el presente estudio de investigación, el cual se presenta a continuación, y cuyos objetivos generales a alcanzar se pueden declarar en los siguientes términos:

- Conocer si existen diferencias significativas en función del género de los estudiantes respecto a las percepciones que tienen en relación al trabajo en grupo e individual.

- Conocer si existen diferencias significativas en función de la edad de los estudiantes, respecto a las percepciones que tienen en relación al trabajo en grupo e individual.

Redes sociales y Tecnologías de la Información y la Comunicación en Educación: aprendizaje colaborativo, diferencias de género, edad y preferencias. María del Carmen Llorente, Julio Cabero y Julio Barroso. 
- Determinar qué herramientas de software social utilizan preferentemente los alumnos.

- Si el uso de estas herramientas de software social depende de la edad, el género, y el país donde cursan los estudios.

\subsection{Fases de desarrollo de la investigación}

De acuerdo con Bisquerra (2004) y Arnal et al. (1992), el estudio que se presenta se puede enmarcar en uno de tipo descriptivo: “... tienen como principal objetivo conocer los cambios que se producen en los sujetos con el transcurso del tiempo; las distintas matizaciones del método de encuesta orientadas a la descripción de una situación dada; el estudio de casos, más centrados en describir y analizar detalladamente unidades o entidades educativas únicas; y el método observacional, caracterizado porque la información es recogida de forma directa de los sujetos observados y no mediante sus respuestas". Y, más concretamente, en los denominados "ex post facto", que son aquellos en los que el investigador se plantea la validación de las hipótesis cuando el fenómeno ya ha sucedido (Bisquerra, 2004), o se encuentra en fase de desarrollo. Dicho en otros términos, únicamente se recogerá información, y no se introducirá ni manipulará ninguna variable.

Apuntar que, por otro lado, en este estudio únicamente se empleará una técnica de recogida de información bajo la adaptación del cuestionario elaborado por Anderson et al. (2009) y denominado "Social Software survey used with unpaced undergrad".

\subsection{Muestra}

El muestreo realizado es de tipo intencional, es decir, los datos que se han encontrado responden al deseo de participar de los estudiantes que cumplimentaron el cuestionario, y al acceso que los diferentes profesores de los distintos países tenían a los alumnos.

El número de participantes fue de 1.040, pertenecientes a universidades de España (Sevilla, País Vasco y Córdoba), Venezuela (Metropolitana y Carabobo), República Dominicana (PUCAIMA) y Argentina (UTN). El 70,00\% eran mujeres, que atendiendo a las diferentes universidades -salvo en la Universidad de Sevilla $(67,47 \%$ mujeres versus $32,53 \%$ hombres)- era homogénea en su distribución. Con respecto a la edad, la gran mayoría se situaba en el intervalo 17-20 años (45,65\%), seguido de 21-24 años $(26,99 \%)$ y de $25-28$ años $(11,67 \%)$, aunque en la República Dominicana destacaba el intervalo 25-28 años (56,19\%), y en Argentina el de 21-24 años y 25-28 años, con un porcentaje del $28,92 \%$ respectivamente.

\subsection{Instrumentos de recogida de información: construcción y fiabilización.}

El instrumento del que se ha partido para llevar a cabo el trabajo es, tal como se apuntaba anteriormente, el elaborado por Anderson et al. (2009) denominado "Social Software survey used with unpaced undergrad", conformado originalmente por 91 ítems distribuidos en las siguientes grandes dimensiones: Identificación / Preferencia de

Redes sociales y Tecnologías de la Información y la Comunicación en Educación: aprendizaje colaborativo, diferencias de género, edad y preferencias. María del Carmen Llorente, Julio Cabero y Julio Barroso.

Página 11 de 23 
Aprendizaje / Habilidades técnicas-tecnológicas / Experiencia en Software Social / Software Social para el Aprendizaje / Confianza en las habilidades / Conclusiones.

En este caso, por los objetivos que se perseguían, se ha realizado una serie de transformaciones, tales como: incorporación de una nueva pregunta destinada a identificar las diferentes Universidades que iban a formar parte de la investigación, y por otra, se eliminó las dos últimas dimensiones del cuestionario original, ya que no se adecuaban a los objetivos que se perseguían en el trabajo.

Así pues, el instrumento quedó conformado por 68 ítems y constaba de diferentes partes: aspectos generales para la identificación de algunas características de la persona que lo cumplimentaba (género, edad, si ha realizado acciones formativas a través de internet,...) (6 ítems), preguntas para indagar si prefería trabajar en grupo o de forma individual (27 ítems), preguntas para conocer las habilidades técnicas tecnológicas (15 ítems), preguntas sobre su experiencia en el trabajo con diferentes herramientas de la denominada web 2.0 (10 ítems), y uso de distintas herramientas de software social en los cursos de formación a distancia (9 ítems). Su administración se llevó a cabo vía Internet durante el otoño de 2012.

\subsection{Técnicas para el análisis de los datos.}

Para la realización de los análisis estadísticos se empleó el programa SPSS, bajo Windows, donde los análisis efectuados quedaron esquematizados en la Tabla 1, así como los objetivos que se han pretendido alcanzar con cada uno de ellos.

\begin{tabular}{|l|l|}
\hline \multicolumn{1}{|c|}{ Objetivos } & \multicolumn{1}{|c|}{$\begin{array}{c}\text { Test/contrastes estadísticos } \\
\text { utilizados }\end{array}$} \\
\hline $\begin{array}{l}\text { - Análisis global de los } \\
\text { resultados. Descripción de las } \\
\text { opiniones de los profesores en los } \\
\text { diferentes ítems formulados. }\end{array}$ & $\begin{array}{l}\text { - Medias, desviaciones típicas y } \\
\text { porcentajes. }\end{array}$ \\
\hline $\begin{array}{l}\text { - Fiabilidad de diferentes ítems } \\
\text { del cuestionario. }\end{array}$ & $\begin{array}{l}\text { Coeficiente de consistencia } \\
\text { interna } \propto \text { de Cronbach. } \\
\text { Coeficiente de correlación ítem- } \\
\text { total instrumento. }\end{array}$ \\
\hline $\begin{array}{l}\text { - Existencia de diferencias entre } \\
\text { distintos tipos de variables. }\end{array}$ & $\begin{array}{l}\text { Whitney, Kruskal-Wallis, y test } \\
\text { de Dunn. }\end{array}$ \\
\hline
\end{tabular}

Tabla 1. Técnicas de análisis utilizadas y objetivos asignados.

Redes sociales y Tecnologías de la Información y la Comunicación en Educación: aprendizaje colaborativo, diferencias de género, edad y preferencias. María del Carmen Llorente, Julio Cabero y Julio Barroso. 


\subsection{Resultados de la investigación.}

De la recogida y análisis de datos, y más concretamente, comenzando por el primero de los objetivos de nuestro estudio (que se refería a conocer si habían diferencias significativas entre el género y las cuatro dimensiones que conformaban el cuestionario: "preferencia de aprendizaje", "habilidades técnicas-tecnológicas", "experiencia software social" y "software social para el aprendizaje"), se formularon para su comprobación las siguientes hipótesis:

H0 (hipótesis nula): no hay diferencias significativas entre el género y las cuatro grandes dimensiones que constituían el cuestionario: "preferencia de aprendizaje", "habilidades técnicas-tecnológicas", "experiencia software social" y "software social para el aprendizaje", con un riesgo alfa de equivocación del 0,05 o inferior.

H1 (hipótesis alternativa): si hay diferencias significativas entre el género y las cuatro grandes dimensiones que constituían el cuestionario: "preferencia de aprendizaje", "habilidades técnicas-tecnológicas", "experiencia software social" y "software social para el aprendizaje", con un riesgo alfa de equivocación del 0,05 o inferior.

El estadístico utilizado en esta ocasión fue la U de Mann-Whitney (Siegel, 1976), prueba no paramétrica aplicada a dos muestras independientes, realizado bajo el programa SPSS. En la Tabla 2 se presentan los resultados alcanzados.

\begin{tabular}{|c|c|c|c|c|c|c|c|}
\hline Dimensiones & $\mathbf{N}$ & $\begin{array}{c}\text { U de } \\
\text { Mann- } \\
\text { Whitney }\end{array}$ & $\begin{array}{c}\text { W de } \\
\text { Wilcoxon }\end{array}$ & $\begin{array}{c}\text { Prob. } \\
\text { Estadística }\end{array}$ & $\begin{array}{l}\text { Error } \\
\text { típico }\end{array}$ & $\begin{array}{c}\text { Estadística } \\
\text { estandarizada }\end{array}$ & Sig. \\
\hline $\begin{array}{c}\text { Preferencias } \\
\text { de } \\
\text { aprendizaje }\end{array}$ & 1.038 & $111.822,500$ & $375.723,500$ & $111.822,500$ & $4.421,508$ & $-0,324$ & 0,746 \\
\hline $\begin{array}{l}\text { Habilidades } \\
\text { Técnicas } \\
\text { Tecnológicas }\end{array}$ & 1.018 & $81.804,000$ & $337.059,000$ & $81.804,000$ & $4.291,194$ & $-6,228$ & $\begin{array}{c}0,000 \\
(* *)\end{array}$ \\
\hline $\begin{array}{c}\text { Experiencia } \\
\text { software } \\
\text { social }\end{array}$ & 1.013 & $90.173,000$ & $342.578,000$ & $90.173,000$ & $4.259,376$ & $-4,083$ & $\begin{array}{c}0,000 \\
(* *)\end{array}$ \\
\hline $\begin{array}{c}\text { Software } \\
\text { social para el } \\
\text { aprendizaje }\end{array}$ & 1.007 & $116.823,500$ & $366.394,500$ & $116.823,500$ & $4.216,374$ & 2,507 & $\begin{array}{c}0,012 \\
(* *)\end{array}$ \\
\hline
\end{tabular}

Tabla 2. Estadístico $U$ de Mann-Whitney (nota: *=significativas al 0,05 y $* *=$ significativas al 0,01$)$.

Los resultados encontrados permiten rechazar la H0 formulada para las dimensiones de "habilidades técnicas-tecnológicas", "experiencia software social" y "software social

Redes sociales y Tecnologías de la Información y la Comunicación en Educación: aprendizaje colaborativo, diferencias de género, edad y preferencias. María del Carmen Llorente, Julio Cabero y Julio Barroso. 
para el aprendizaje", con un riesgo alfa de equivocarnos del 0,01 ; y por tanto, se puede aceptar la $\mathrm{H} 1$, que indica que existen diferencias en función del género en las diferentes dimensiones apuntadas con un riesgo alfa de equivocación del 0,05 o inferior. Por el contrario, no se rechaza la $\mathrm{H} 0$ en lo que hace referencia a las "preferencias de aprendizaje", donde se ha encontrado puntuaciones estadísticamente similares en función del género de los alumnos.

Señalar que las puntuaciones medias más altas para las diferentes dimensiones se dieron en los Hombres, con las siguientes puntuaciones: "habilidades técnicas-tecnológicas" (Hombres 4,0166 - Mujeres 3,7655), y "experiencia social del software" (Hombres 2,5096 - Mujeres 2,3386). Por el contrario, las mujeres puntuaron más alto que la dimensión de "software social para el aprendizaje (Hombres 3,0475 - Mujeres 3,1571). En síntesis, se podría decir que en los dos primeros casos los hombres estaban "más totalmente de acuerdo" y tendían a percibir con niveles más "expertos" que las mujeres; por el contrario, las mujeres se llegaron a considerar "más interesadas en la formación" que los hombres.

Para el siguiente de los objetivos, conocer si había diferencias significativas en las cuatro grandes dimensiones que conforman el estudio y la edad de los estudiantes, al igual que en los casos anteriores, se establecieron las siguientes hipótesis:

H0 (hipótesis nula): no hay diferencias significativa entre la edad de los estudiantes y las cuatro grandes dimensiones que constituían nuestro cuestionario: "preferencia de aprendizaje", "habilidades técnicas-tecnológicas", "experiencia software social" y "software social para el aprendizaje", con un riesgo alfa de equivocación del 0,05 o inferior.

H1 (hipótesis alternativa): si hay diferencias significativa entre la edad de los estudiantes y las cuatro grandes dimensiones que constituían nuestro cuestionario: "preferencia de aprendizaje", "habilidades técnicas-tecnológicas", "experiencia software social" y "software social para el aprendizaje", con un riesgo alfa de equivocación del 0,05 o inferior.

El estadístico que se empleó para ello fue la prueba de Kruskal-Wallis (Gibbons y Chakraborti, 2003) para muestras independientes, realizado bajo el programa SPSS. En la Tabla 3 se presentan los resultados alcanzados.

\begin{tabular}{|l|c|c|c|c|}
\hline & N & K-Wallis & gl & Nivel significación \\
\hline Preferencia de aprendizaje & 1043 & 33,404 & 3 & $0,000(* *)$ \\
\hline Habilidades técnicas-tecnológicas & 1022 & 13,867 & 3 & $0,003(* *)$ \\
\hline Experiencia software social & 1017 & 21,856 & 3 & $0,000(* *)$ \\
\hline Software social para el aprendizaje & 1012 & 5,859 & 3 & 0,119 \\
\hline
\end{tabular}

Tabla 3. Estadístico de Kruskal-Wallis (nota: *=significativas al 0,05 y $* *=$ significativas al 0,01 ).

Redes sociales y Tecnologías de la Información y la Comunicación en Educación: aprendizaje colaborativo, diferencias de género, edad y preferencias. María del Carmen Llorente, Julio Cabero y Julio Barroso. 
Los resultados encontrados permiten rechazar la hipótesis nula $(\mathrm{H} 0)$ formulada para las dimensiones de "preferencias de aprendizaje", "habilidades técnicas-tecnológicas" y "experiencia software social"; en todos los casos con un riesgo alfa de equivocación del 0,05 . Por el contrario no se rechaza la $\mathrm{H} 0$, en lo que hace referencia al "software social para el aprendizaje".

Con el objeto de conocer entre qué parejas de edad se daban las diferencias cuando se había rechazado la H0, se aplicó el test de Dunn (Dunn, 1964) para las pruebas estadísticas de comparaciones múltiples de Kruskal-Wallis, obteniendo las siguientes puntuaciones.

\begin{tabular}{|c|c|c|c|c|}
\hline Muestras & $\begin{array}{c}\text { Prueba } \\
\text { estadística }\end{array}$ & $\begin{array}{l}\text { Error } \\
\text { típico }\end{array}$ & $\begin{array}{l}\text { Des. Prueba } \\
\text { estadística }\end{array}$ & Sig \\
\hline \multicolumn{5}{|c|}{ Preferencias de aprendizaje } \\
\hline $17-20-25-28$ & $-5,624$ & 30,559 & $-0,184$ & 1,000 \\
\hline $17-20-21-24$ & $-57,274$ & 22,634 & $-2,530$ & 0,068 \\
\hline 17-20-Más de 28 & $-83,078$ & 27,268 & $-3,047$ & $\begin{array}{c}0,014 \\
(* *)\end{array}$ \\
\hline $25-28-21-24$ & 51,649 & 32,626 & 1,583 & 0,680 \\
\hline 25-28-Más de 28 & $-77,454$ & 35,996 & $-2,152$ & 0,189 \\
\hline 21-24-Más de 28 & $-25,805$ & 29,566 & $-0,873$ & 1,000 \\
\hline \multicolumn{5}{|c|}{ Habilidades técnicas-tecnológicas } \\
\hline $17-20-25-28$ & $-103,070$ & 30,366 & $-3,394$ & $0,004(* *)$ \\
\hline $17-20-21-24$ & $-25,992$ & 22,356 & $-1,163$ & 1,000 \\
\hline 17-20-Más de 28 & 16,450 & 27,247 & 0,604 & 1,000 \\
\hline $25-28-21-24$ & $-77,078$ & 32,450 & $-2,375$ & 0,105 \\
\hline 25-28-Más de 28 & 119,520 & 35,995 & 3,320 & $0,005(* *)$ \\
\hline 21-24-Más de 28 & 42,442 & 29,552 & 1,436 & 0,906 \\
\hline \multicolumn{5}{|c|}{ Experiencia software social } \\
\hline $17-20-25-28$ & 8,904 & 30,201 & 0,295 & 1,000 \\
\hline $17-20-21-24$ & 11,651 & 22,261 & 0,523 & 1,000 \\
\hline
\end{tabular}

Redes sociales y Tecnologías de la Información y la Comunicación en Educación: aprendizaje colaborativo, diferencias de género, edad y preferencias. María del Carmen Llorente, Julio Cabero y Julio Barroso. 


\begin{tabular}{|c|c|c|c|c|}
\hline Muestras & $\begin{array}{c}\text { Prueba } \\
\text { estadística }\end{array}$ & $\begin{array}{c}\text { Error } \\
\text { típico }\end{array}$ & $\begin{array}{c}\text { Des. Prueba } \\
\text { estadística }\end{array}$ & Sig \\
\hline 17-20-Más de 28 & 124,742 & 27,366 & 4,558 & 0,000 \\
$(* *)$
\end{tabular}

Tabla 4. Diferencias significativas entre parejas de edad en las diferentes dimensiones analizadas en el instrumento (nota: $*=$ significativas al $0,05 \mathrm{y} * *=$ significativas al 0,01 ).

En la primera de las dimensiones, "preferencias de aprendizaje", las diferencias significativas se han encontrado entre los alumnos con edades comprendidas en la pareja de edades de 17-20 años y más de 28 años. Para una correcta interpretación de los datos, se debe señalar que las puntuaciones medias más altas, y que indicaban por lo tanto percepciones de niveles más altos entre la edad de los sujetos y las preferencias de aprendizaje, se dieron en las siguientes franjas de edades: 25-28 años $(3,46030)$, seguidas de las edades comprendidas entre 17-20 años (3,4449), más de 28 años $(3,5433)$, y por último, los alumnos de edades comprendidas entre 21-24 años $(3,51116)$, siendo por tanto los alumnos de más edad los que se mostraban más de acuerdo con las preferencias hacia el trabajo grupal.

Respecto a la segunda de las dimensiones "habilidades técnico-tecnológicas" las diferencias significativas se han dado entre los estudiantes con edades comprendidas entre las franjas de edad 17-20 años y 25-28 años y la pareja de edades comprendidas entre más de 28 años y 25-28 años. Los valores medios alcanzados por los estudiantes según sus edades fueron los siguientes: el valor más alto fue para los estudiantes con edades comprendidas entre los 21-24 años $(3,8674)$, seguidos por los de edades comprendidas entre $17-20$ años $(3,8140)$, de más de 28 años $(3,7586)$ y por último los de la franja de edades 25-28 (3,9934).

En lo que se refiere a la "experiencia software social", las diferencias se dieron entre estudiantes con edades comprendidas en las siguientes parejas de edad: más de 28-21-24 años; más de 28-25-28 años y más de 28-17-20 años. En este caso las puntuaciones medias más altas fueron las obtenidas por los alumnos con edades comprendidas entre los 21-24 años (2,4189), seguido de las de edades comprendidas entre 17-20 años $(2,4284)$, más de $28(2,1750)$ y 25-28 años $(2,4291)$. Recuérdese que cuanto más elevadas sean las puntuaciones más expertos se consideraban los alumnos hacia el ámbito de la tecnología.

Por último, los resultados obtenidos en el estudio del objetivo "Determinar qué herramientas de software social utilizan preferentemente los alumnos", una de las preguntas del cuestionario se centraba en que nos informaran de la red (Facebook, Twiter, Linkedin y Hi5) que fundamentalmente utilizaban, y al respecto se encontró que

Redes sociales y Tecnologías de la Información y la Comunicación en Educación: aprendizaje colaborativo, diferencias de género, edad y preferencias. María del Carmen Llorente, Julio Cabero y Julio Barroso. 
el 66,34\% (f=613) indicó Facebook y el 31,60\% (f=292) Twiter. Señalar que las otras dos opciones ofrecidas en su cuestionario, Linkedin y Hi5, obtuvieron un porcentaje de respuesta cercana al $2 \%$. Más específicamente, los resultados alcanzados por países con respecto a la preferencia de redes sociales y las más utilizadas, se presentan en la Tabla 5.

\begin{tabular}{|l|c|c|c|c|c|c|c|c|}
\hline & \multicolumn{2}{|c|}{ España } & \multicolumn{2}{|c|}{ República Dominicana } & \multicolumn{2}{|c|}{ Argentina } & \multicolumn{2}{|c|}{ Venezuela } \\
\cline { 2 - 9 } & $\mathrm{f}$ & $\%$ & $\mathrm{f}$ & $\%$ & $\mathrm{f}$ & $\%$ & $\mathrm{f}$ & $\%$ \\
\hline Facebook & 205 & 45,25 & 74 & 84,09 & 125 & 84,46 & 207 & 88,84 \\
\hline Twiter & 245 & 54,08 & 14 & 15,91 & 8 & 5,41 & 25 & 10,73 \\
\hline Linkedin & 1 & 0,22 & 0 & 0,00 & 15 & 10,14 & 1 & 0,43 \\
\hline Hi5 & 2 & 0,44 & 0 & 0,00 & 0 & 0,00 & 0 & 0,00 \\
\hline
\end{tabular}

Tabla 5. Red qué más utiliza.

\section{Conclusiones.}

A la luz de los resultados obtenidos con el desarrollo de la investigación apuntar como los estudiantes, independientemente del país latinoamericano donde cursan sus estudios, tienen una actitud positiva para el trabajo colaborativo o en grupo, y consideran que este tipo de acciones formativas no tienen por qué repercutir de forma negativa en los resultados de aprendizaje que alcancen. Ello hace posible contemplar que, inicialmente, existen actitudes y predisposiciones significativas a trabajar desde esta perspectiva metodológica, por ello el docente universitario no debe tener miedo ni recelos a su incorporación en los procesos de formación virtual. Por otra parte, es importante tener en cuenta esta predisposición, pues determinará, en gran medida, la viabilidad de la aplicación de este tipo de acciones. Estas predisposiciones positivas posiblemente vengan determinas por diferentes tipos de causas, que quedan establecidas en los siguientes términos: a) el haber tenido distintas experiencias positivas en las actividades organizadas alrededor del trabajo en grupo, para alcanzar resultados significativos de aprendizaje a lo largo de su período formativo, y b) que perciben, independientemente del país en el cual cursan sus estudios, que el trabajo en grupo es una de las formas de trabajar más usuales y productivas en la sociedad del conocimiento.

De la investigación realizada también se desprende que los alumnos tienen una fuerte experiencia en el trabajo educativo basado en Internet, de ahí que experiencias de formación siguiendo esta tecnología puedan tener buena acogida entre los estudiantes, y sea un hecho constatable el que desde las instituciones de enseñanza superior se pueda trabajar en el contexto hispanoamericano en lo que se denomina movilidad virtual de los estudiantes para el aprendizaje (García Aretio, 2008).

Redes sociales y Tecnologías de la Información y la Comunicación en Educación: aprendizaje colaborativo, diferencias de género, edad y preferencias. María del Carmen Llorente, Julio Cabero y Julio Barroso. 
El gran interés por el trabajo en grupo se percibe también por la opinión mostrada por los estudiantes, que perciben como su utilización no tiene por qué conllevar la obtención de resultados inferiores en la práctica educativa, y que su utilización les brinda una gran posibilidad de soporte y ayuda para aprender.

Respecto a las actitudes que los alumnos muestran hacia las TIC y las Redes Sociales, hemos encontrado que tienen actitudes significativas hacia las mismas, lo que coincide con los hallazgos de otros autores (Sampedro y Marín, 2015; Cruz, 2016), y ello además es independiente del país en el cual cursan sus estudios. Ahora bien, en este apartado de las TIC, los datos encontrados nos llevan a señalar que los alumnos no son tan "nativos" en su dominio como desde ciertos sectores se ha hecho creer. Los alumnos han señalado que se encuentran capacitados en el uso de las redes sociales, uso de sitios para compartir vídeos en la red, o referidos a la publicación de fotografías; en contrapartida, han señalado que no presentan fuerte formación en otras TIC como los blog, las wikis, las videoconferencias o los marcadores sociales, y ello es una constante independientemente del país en el cual el estudiante cursa sus estudios.

Las redes que los alumnos principalmente utilizan son Facebook y Twiter, lo que permiten apuntar que las experiencias que en los últimos tiempos se están llevando a cabo para su incorporación en el terreno educativo (Duffy, 2011; Llorens y Capdeferro, 2011; Túñez y García, 2012; Web-Long y Chun-Yi, 2013; Cruz, 2016), cuentan con la ventaja inicial del conocimiento que los alumnos tienen respecto a las mismas.

Por otro lado, también se han encontrado diferencias significativas respecto al género, aunque hay que señalar que no se encontraron respecto a sus preferencias para el trabajo en grupo. En general, las mujeres presentaron unas puntuaciones más altas en las percepciones que tenían en estar más interesadas en movilizar las diferentes herramientas de la web 2.0. En contrapartida, los hombres lo estaban, presentando mayores habilidades técnicas-tecnológicas respecto a las diferentes herramientas que se les ofrecieron, así como que tenían un nivel más avanzado o experto en la utilización de las mismas. Resultados que coinciden con los alcanzados por otros autores respecto al uso y valoración que hacen los alumnos respecto a las tecnologías (Lim y Meier, 2011; Hohlfeld, Ritzhaupt, y Barron, 2013; Cruz, 2016).

El que los estudiantes hubieran realizado acciones formativas previas a través de Internet resultó ser un condicionante en cuanto a las habilidades técnicas-tecnológicas que tenían respecto a diferentes tecnologías, la experiencia que tenían respecto a diferentes tecnologías, y lo interesados que estaban respecto a las mismas, resultado que coincide con los obtenidos por otros autores (Kennedy, Judd, Dalgarno, y Waycott, 2010; Sánchez, Muñoz y Paredes, 2015), y que nos lleva de nuevo a reclamar la necesidad de llevar a cabo en los centros la alfabetización digital de los estudiantes para que adquieran una fuerte competencia digital (Cabero, Marín, y Llorente, 2012).

Presentación del artículo: 15 de Julio de 2016

Fecha de aprobación: 15 de Octubre de 2016

Fecha de publicación: 15 de Noviembre de 2016

Redes sociales y Tecnologías de la Información y la Comunicación en Educación: aprendizaje colaborativo, diferencias de género, edad y preferencias. María del Carmen Llorente, Julio Cabero y Julio Barroso.

Página 18 de 23 
Cabero, J., Barroso, J. Llorente, M.C. y Yanes, C. (2016). Redes Sociales y Tecnologías de la Información y la Comunicación: diferencias de género, edad y preferencias. RED. Revista de Educación a Distancia, 51. Consultado el (dd/mm/aaaa) en http://www.um.es/ead/red/

\section{Bibliografía}

Abramson, L. (2011). Can social networking keep students in school? NPR: Morning Edition. Recuperado de http://www.npr.org/2011/02/09/133598049/can-social-networking-keep-studentsin-school

Ajjan, H., y Hartshorne, R. (2008). Investigating faculty decisions to adopt Web 2.0 technologies: theory and empirical tests. The Internet and Higher Education, 11(2), 71-80.

Anderson, T., Poellhuber, B., y Mckerlich, R. (2010). Social Software survey used with unpaced undergrad. Recuperado de http://auspace.athabascau.ca/handle/2149/2771

Arnal, J.; Del Rincón, D., y Latorre, A. (1992). Investigación educativa. Fundamentos y metodología. Barcelona: Labor.

Arnold, N., y Trena, P. (2010). Using a social networking site for experiential learning: Appropiating, lurking, modeling and community building. Internet and Higher Education, 13(4), 188-196.

Aydin, S. (2012). A review of research on Facebook as an educational environment. Education Tech Research Development, 60(6), 1093-1106.

Bartlett-Bragg, A. (2006), Reflections on pedagogy: reframing practice to foster informal learning with social software. Recuperado de http://www.dream.sdu.dk/uploads/_les/Anne\%20Bartlett-Bragg.pdf

Barron, Br. (2006). Interest and self-sustained learning as catalysts of development: A learning ecologies perspective. Human Development, 49, 193-224.

Bisquerra, R. (2004). Metodología de la investigación educativa. Madrid: La Muralla.

Boyd, S. (2005). Are You Ready for Social Software? Recuperado de http://www.stoweboyd.com/message/2006/10/are_you_ready_f.html

Boyd, D. y Ellison, N. (2008). Social network sites: definition, history, and scholarship. Journal of Computer-Mediated Communication, 13(1). Recuperado de http://jcmc.indiana.edu/vol13/issue1/boyd.ellison.html

Cabero, J. (2003). Principios pedagógicos, psicológicos y sociológicos del trabajo colaborativo su proyección en la tele-enseñanza, en Martínez, Redes de comunicación en la enseñanza. Barcelona: Paidós.

Redes sociales y Tecnologías de la Información y la Comunicación en Educación: aprendizaje colaborativo, diferencias de género, edad y preferencias. María del Carmen Llorente, Julio Cabero y Julio 
Cabero, J. (2006). Comunidades virtuales para el aprendizaje. Su utilización en la enseñanza. EDUTEC. Revista Electrónica de Tecnología Educativa, 20. Recuperado de http://edutec.rediris.es/Revelec2/revelec20/cabero20.htm

Cabero, J. (2012). Las redes sociales en el entramado educativo de la Web. 2.0. En E. Navas, Web 2.0 Innovación e investigación educativa. Caracas: Univ. Metropolitana, pp. 21-28.

Cabero, J. y Llorente, M.C. (2010). Comunidades virtuales para el aprendizaje, EDUTEC. Revista Electrónica de Tecnología Educativa, 34, 1-10.

Cabero, J., Marín, V. \& Llorente, M.C (2012). Desarrollar la competencia digital. Educación mediática a lo largo de toda la vida. Sevilla: Eduforma

Cabero, J., y Marín, V. (2013). Percepciones de los estudiantes universitarios latinoamericanos sobre las redes sociales y el trabajo en grupo. RUSC. Universities and Knowledge Society Journal, 10(2), 219-235.

Cachia, R. (2008). Los sitios de creación de redes. Aspectos Sociales. Revista Telos (76). Recuperado de http://telos.fundaciontelefonica.com/telos/articulocuaderno.asp@idarticulo=4\&rev= 76.htm

Camacho, M. (2010). Las redes sociales para enseñar y aprender. Reflexiones pedagógicas básicas. En L. Castañeda, Aprendizajes con redes sociales. Tejidos educativos para los nuevos entornos. Sevilla: Eduforma, pp. 91-104

Cruz, I. (2016). Percepciones en el uso de las redes sociales y su aplicación en la enseñanza de las matemáticas. Pixel-Bit. Revista de Medios y Educación, 48.

Davis, F. (1989). Perceived usefulness, perceived ease of use, and user acceptance of information technology. MIS Quarterly, 13(3), 319-340.

De Haro, J. (2010). Redes Sociales para la educación . Madrid: Anaya.

Domínguez, G. y Llorente, M.C. (2009). La educación social y la Web 2.0. Nuevos espacios de innovación e interacción social en el Espacio Europeo de Educación Superior. Pixel-Bit. Revista de Medios y Educación, 35, 105-114.

Duffy, P. (2011). Facebook or faceblock: cautionary tales exploring the rise of social networking, in C. McLoughlin, (comps.), Web 2.0. Based e-learning, New York: Information Science Reference.

Dunn, O. (1964). Multiple Comparisons Using Rank Sums. Technometrics, Vol. 6, pp. 241-241.

Durall, E., Gros, B., Maina, M., Johnson, L., y Adams, S. (2012). Perspectivas tecnológicas: educación superior en Iberoamérica 2012-2017. Austin, Texas: The New Media Consortium.

García Aretio, L. (2008). Netactive: Bases y propuestas para las buenas prácticas en movilidad virtual (Un enfoque intercontinental). Madrid: UNED.

Redes sociales y Tecnologías de la Información y la Comunicación en Educación: aprendizaje colaborativo, diferencias de género, edad y preferencias. María del Carmen Llorente, Julio Cabero y Julio Barroso.

Página 20 de 23 
Güzin, S., y Koçak, Y. (2010). Modeling educational usage of Facebook. Computers \& Education, 55, 444-455.

Hannafin, M., Land, S., y Oliver, K. (1999). Open learning environments: Foundations, methods, and models, in C. Reigeluth (Ed.), Instructional Design Theories and Models. Mahwah, N. J., Lawrence Erlbaum, Association.

Hargittai, E. (2008). Whose space? Differences among users and not-users of social network sites. Journal of Computer-Mediated Communication, 13(1), 276-297.

Jones, S., y Fox, S. (2009). Generations online in 2009. Data memo. Washington, DC: Pew Internet and American Life Project, Recuperado de http://www.pewinternet.org/w/media//Files/Reports/2009/PIP_Generations_2009.p $\underline{\mathrm{df}}$

Kapp, K., y O'driscoll, T. (2010). Learning in 3D. San Francisco: CA, Pfeiffer.

Kirschner, P., y Kirpinski, A. (2010). Facebook and academic performance. Computer in Human Behaviors, 26, 1237-1245.

Lee, M. y Mcloughlin, C. (2008). Harnessing the affordances of Web 2.0 and social software tools: can we naturally make "student-centered" learning a reality?. Paper presented at the World Conference on Educational Multimedia, Hypermedia and Telecommunications. Vienna: Austria.

Llorens, F., y Capdeferro, N. (2011). Posibilidades de la plataforma Facebook para el aprendizaje colaborativo en línea. RUSC, Revista de Universidad y Sociedad del Conocimiento, 8(2), 31-45.

Llorente, M.C. (2013). Assessing personal learning environments (PLEs). An expert evaluation". New Approaches in Educational Research, 2(1), 39-44, Recuperado de http://naerjournal.ua.es/article/view/v2n1-6

Llorente, M.C. (2013). Aprendizaje autorregulado y PLE. Edmetic. Revista de Educación Mediática y TIC, 2(1), 63-79, Recuperado de http://www.edmetic.es/revistaedmetic/index.php/component/content/article?id=45

López, E., y Ballesteros, C. (2008). Caminando hacia el software social: una experiencia universitaria con blogs. Pixel-Bit. Revista de Medios y Educación, 32, 67-82.

López, E., Vásquez, E., y Sánchez-Serrano, J. L. (2015). Estudio bibliométrico de Pixelbit, revista de medios y educación (2000-2013). Pixel-Bit. Revista de Medios y Educación, 46, 65-85.

Martínez, F. (2003). Redes de comunicación en la enseñanza. Barcelona: Paidós.

Martín-Moreno, E. (2004). Aprendizaje colaborativo y redes de conocimiento. Actas de las IX Jornadas Andaluzas de Organización y Dirección de Instituciones Educativas. Granada: Grupo Editorial Universitario.

Mason, R. (2006). Learning technologies for adult continuing education. Studies in Continuing Education, Vol. 28, n 2, pp. 121-133.

Mason, R., y Rennie, F. (2008). E-learning and social networking handbook. London:

Redes sociales y Tecnologías de la Información y la Comunicación en Educación: aprendizaje colaborativo, diferencias de género, edad y preferencias. María del Carmen Llorente, Julio Cabero y Julio Barroso.

Página 21 de 23 
Routledge.

Maxwell, J. (2001). Las 17 leyes incuestionables del trabajo en equipo. Estados Unidos: Caribe.

O'Keeffe, G., y Clarke, K. (2011). The impact of social media on children, adolescents, and families. Pediatrics, 127, 800-804.

Parameswaran, M., y Whinston, A. (2007). Social computing: an overview. Communications of the Association for Information Systems, 19, 762-780.

Roblyer, M., Mcdaniel, M., Webb, M., Herman, J., y Vince, J. (2010). Findings on Facebook in higher education: a comparison of college faculty and student uses and perceptions of social networking sites. Internet and Higher Education, 13(3), 134140.

Rodríguez Illeras, J.L. (2007). Comunidades virtuales, prácticas y aprendizaje: elementos para una problemática. Revista Electrónica de la Educación, Educación y Cultura en la Sociedad de la Información, 8(3), 6-22.

Roig, R. (2011). Redes Sociales educativas. Propuestas para la intervención en el aula. En F. Martínez, \& I. Solano, Comunicación y relaciones sociales de los jóvenes en la red. Murcia, Marfil, pp. 137-151.

Rogers, E. (2003). Diffusion of innovation. New York: Free Press.

Romero, J. (2011). Las redes sociales en educación. Revista digital CIS-F. Recuperado de http://www.csif.es/archivos/andalucia/ensenanza/revistas/iee/Numero_44/JOSE_LUIS_ROMERO _LACAL_1.pdf

Sánchez, P., Muñoz, T., y Paredes, J. (2015). El trabajo en el aula y la competencia digital en el modelo 1 a 1 de la Comunidad de Madrid. Pixel-Bit. Revista de Medios y Educación, 47, pp. 41-58.

Salinas, J. (2004). Comunidades virtuales de aprendizaje. Comunicación y Pedagogía, Vol. 194, pp. 20-24.

Sampedro, B., y Marín, V. (2015). Conocimiento de los futuros educadores sociales de las herramientas web 2.0. Pixel-Bit. Revista de Medios y Educación, 47, pp. 211222.

Santamaría, F. (2008). Posibilidades pedagógicas. Redes sociales y comunidades pedagógicas. TELOS. Cuadernos de innovación y comunicación, 76.

Siemens, G. (2005). Connectivism: a learning theory for the digital age. Elearnspace. Recuperado de http://www.elearnspace.org/Articles/connectivisim.htm

Túñez, M., y García, J. (2012). Las redes sociales como entorno docente: análisis del uso de Facebook en la docencia universitaria. Pixel Bit, Revista de Medios y Educación, 41, 77-92.

Redes sociales y Tecnologías de la Información y la Comunicación en Educación: aprendizaje colaborativo, diferencias de género, edad y preferencias. María del Carmen Llorente, Julio Cabero y Julio Barroso.

Página 22 de 23 
Vygotsky, L. (1978). Mind and society: The development of higher mental processe. Cambridge, MA: Harvard University Press.

Web-Long, C., y Chun-Yi, L. (2013). Trust as a learning facilitator that affects students' learning performance in the Facebook community: An investigation in a business planning writing course. Computers \& Education, 62, 320-327.

Redes sociales y Tecnologías de la Información y la Comunicación en Educación: aprendizaje colaborativo, diferencias de género, edad y preferencias. María del Carmen Llorente, Julio Cabero y Julio Barroso.

Página 23 de 23 